\title{
Vegetation Database Forests and Grasslands of the Lower Werra Region
}

\author{
Cord Peppler-Lisbach
}

\begin{abstract}
The Vegetation Database Forests and Grasslands of the Lower Werra Region (GIVD ID EU-DE-011) includes vegetations relevés made within the scope of a project dealing with statistical distribution modelling of land-use types, plant communities and species. The study covers a 10 x $4 \mathrm{~km}$ landscape gradient in Northern Hesse. The plots were chosen with a stratified-random design and have a uniform plot size of $100 \mathrm{~m}^{2}$ (forests) and $25 \mathrm{~m}^{2}$ (grassland), respectively. Apart from numerous site parameters measured in the relevé plots, comprehensive information derived from GIS maps are also available.
\end{abstract}

Keywords: Festuco-Brometea; Hessen; Molinio-Arrhenatheretea; Querco-Fagetea.

GIVD Database ID: EU-DE-011

Last update: 2012-07-07

\section{Vegetation Database Forests and Grasslands of the Lower Werra Region}

Scope: woodland and grassland releves, lower Werra region (Central Germany)

Status: ongoing capture

Period: 2002-2009

Database manager(s): Cord Peppler-Lisbach (cord.peppler.lisbach@uni-oldenburg.de)

Owner: [NA]

Web address: [NA]

Availability: free upon request

Online upload: no

Online search: no

Database format(s): TURBOVEG

Export format(s): SQL, PDF

Publication: [NA]

Plot type(s): normal plots

Plot-size range: $25-100 \mathrm{~m}^{2}$

Non-overlapping plots: 565

Estimate of existing plots: [NA]

Completeness: [NA]

Total plot observations: 565

Number of sources: $[N A]$

Valid taxa: $[N A]$

Countries: DE: $100.0 \%$

Forest: $50 \%$ - Non-forest: aquatic: $0 \%$; semi-aquatic: $0 \%$; arctic-alpine: $0 \%$; natural: $0 \%$; semi-natural: $50 \%$; anthropogenic: $0 \%$

Guilds: all vascular plants: $100 \%$

Environmental data: altitude: $100 \%$; slope aspect: 100\%; slope inclination: 100\%; surface cover other than plants (open soil, litter, bare rock etc.) $100 \%$; soil pH: $100 \%$; other soil attributes: $50 \%$

Performance measure(s): cover: $100 \%$

Geographic localisation: GPS coordinates (precision $25 \mathrm{~m}$ or less): $100 \%$

Sampling periods: $2000-2009: 100.0 \%$

Information as of 2012-07-12; further details and future updates available from http://www.givd.info/ID/EU-DE-011

Cord Peppler-Lisbach (cord.peppler.lisbach@uni-oldenburg.de)

Institut für Biologie und Umweltwissenschaften, Universität Oldenburg, Postfach 2503, 26111 Oldenburg, GERMANY 